\title{
Refusal to Take Sick Leave after Being Diagnosed with a Communicable Disease as an Estimate of the Phenomenon of Presenteeism in Poland
}

\author{
Marcin Mikos ${ }^{\mathrm{a}} \quad$ Grzegorz Juszczyk $^{\mathrm{b}}$ Aleksandra Czerw ${ }^{\mathrm{b}}$

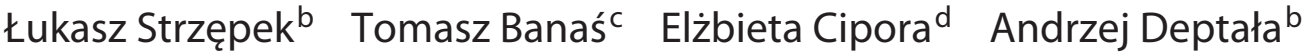 \\ Anna Badowska-Kozakiewicz ${ }^{b}$ \\ ${ }^{a}$ Faculty of Medicine and Health Sciences, Andrzej Frycz Modrzewski Krakow University, Krakow, Poland; \\ ${ }^{\mathrm{b}}$ Medical University of Warsaw, Warsaw, Poland; ${ }^{\mathrm{C} C o l l e g i u m ~ M e d i c u m, ~ J a g i e l l o n i a n ~ U n i v e r s i t y, ~ K r a k o w, ~ P o l a n d ; ~}$ \\ ${ }^{\mathrm{d}}$ Medical Institute, Jan Grodek State Vocational Academy in Sanok, Sanok, Poland
}

\section{Highlights of the Study}

- Absenteeism and presenteeism generate substantial costs for employers.

- Absenteeism was monitored using an objective doctor's assessment and presenteeism was determined by self-reported information from the employee.

- This study analyses doctors' reported refusal to accept sick leave for patients with communicable disease in Poland. This may not only limit the patients' ability to work but also expose other employees.

\section{Keywords}

Presenteeism · Communicable disease · Sick leave

\begin{abstract}
Objective: The purpose of this study was to estimate the prevalence of presenteeism in patients with communicable diseases in Poland. Subject and Methods: This study was based on data from the medical records of 2,529 patients aged 19-64 years. All of the patients were diagnosed with communicable diseases. The inclusion criteria were based on implementing decision concerning communicable diseases made by the Commission of the European Union. Associations between refusal to take sick leave and patients' age, gender, and diagnosis in terms of the International Statistical Classification of Diseases and Related Health Problems (ICD-10) were tested. Linear regression analysis of the
\end{abstract}

data acquired from the patients who agreed to take sick leave was further used to estimate the possible length of sick leave in patients who refused to take it. Results: The number of patients who refused to take sick leave was $18.1 \%$. The presenteeism rate was related to the age of patients (periods of sick leave were longer in older patients) and the ICD-10 diagnosis (largely in bacterial intestinal infections and measles). The estimated number of days spent on sick leave in patients who refused to take it, assuming that they made a different decision and complied with it, was in the range of 4-6 days. Conclusion: The prevalence of presenteeism in the case of communicable diseases in Poland is lower than in the general population. However, as the refusals to take sick leave took place in the case of potentially contagious diseases, the negative impact on productivity may be significant.

(C) 2019 The Author(s)

Published by S. Karger AG, Basel

\begin{tabular}{ll}
\hline KARGER & @ 2019 The Author(s) \\
& Published by S. Karger AG, Basel Openger \\
karger@karger.com & This is an Open Access article licensed under the Creative Commons \\
www.karger.com/mpp & $\begin{array}{l}\text { Attribution-NonCommercial-4.0 International License (CC BY-NC) } \\
\text { (http://www.karger.com/Services/OpenAccessLicense), applicable to } \\
\text { the online version of the article only. Usage and distribution for com- } \\
\text { mercial purposes requires written permission. }\end{array}$
\end{tabular}

Aleksandra Czerw

Medical University of Warsaw Zwirki i Wigury 61

PL-02-091 Warsaw (Poland)

E-Mail aleksandra.czerw@wum.edu.pl 


\section{Introduction}

Two main phenomena related to workers' diseases from the perspective of their professional activity are absenteeism and presenteeism. Absenteeism refers to the period of being professionally inactive due to one's health status, while presenteeism means to be professionally active, i.e., attending work or running a business, despite being ill. Together with the direct costs of health care, both lead to a loss of productivity and are the 3 main categories of employers' costs related to the employee health status. Researchers who have provided an estimate of absenteeism costs and its associations with other variables [1-4], as well as costs of presenteeism, have found that they are higher than the costs of absenteeism $[5,6]$. Estimates on the proportion of the costs of presenteeism range from 18 to $61 \%$ [7]. The costs of presenteeism in terms of a reduced professional efficiency in the USA have been estimated to reach USD 150 billion per year [8]. In a Polish sample of 550,360 patients, $27.4 \%$ refused to take sick leave [9].

Predictors of presenteeism have been investigated. It has been found that if the pace of work is not so important or if it is not controlled then presenteeism is more prevalent [10]. The level of presenteeism is also higher when there is no possibility of replacement and the essential work accumulates until the ill person returns [11]. A sense of loyalty toward colleagues when working in a team also strengthens presenteeism [12]. Presenteeism is negatively correlated with job satisfaction and positively correlated with stress at work and professional burnout $[13,14]$. Being professionally active despite ill health may worsen health and lead to absenteeism [15]. Depending on the type of disease, however, the problem of presenteeism may arise even if absenteeism is not noticeable [16]. Patients suffering from a migraine are less productive, but this does not mean they have a greater number of absences from work.

Presenteeism has its consequences. Both the quantity and the quality of work are at stake. Health conditions as well as medication or treatment may lead to difficulties with concentration, persistent distraction, fatigue, and irritability, which hinder professional effectiveness and productivity. Specific consequences depend on the reason for being ill, which can be acute, episodic, or chronic, and the type of job. Attending work while experiencing minor discomfort after a minor accident, even with a reduced productivity, may be beneficial compared to being absent. Communicable diseases also pose the risk of spreading the illness and, in this case, presenteeism leads not only to a loss of productivity in the people that cope with the disease but also to a loss of productivity and possibly absenteeism in other people who could get infected; however, in most cases, infection precedes the onset of symptoms, which makes it difficult to estimate the possible decrease in the number of infected workers if presenteeism could be reduced.

A theory of presenteeism summarizing the provision of theorems about relationships and possible interactions between variables is being developed [17]. It refers to presenteeism, productivity loss, and personality traits. Attitudes and organizational factors are considered possible moderators.

The prevalence of presenteeism in Poland on the basis of medical records and associations between presenteeism and patients' age, gender, and type of medical problem was presented by Juszczyk et al. [9]. The highest percentage of refusals of taking sick leave was recorded when the International Statistical Classification of Diseases and Related Health Problems (ICD-10) diagnostic codes J01 to J04 were used. The codes refer to acute sinusitis, acute pharyngitis, and acute tonsillitis as well as acute laryngitis and tracheitis. These communicable diseases are acute upper respiratory infections. The current study concerns the cases of refusal to take sick leave after being diagnosed with a communicable disease during a medical examination. The list of diagnoses is based on the Commission Implementing Decision (EU) 2018/945 of June 22, 2018, on communicable diseases [18].

The aim of this study was to estimate the prevalence of presenteeism after being diagnosed with a communicable disease in Poland and to explore possible associations between presenteeism and patients' age, gender, and specific type of medical problem. We hypothesized that: (1) there is an association between the diagnosis and presenteeism, (2) there is association between patients' gender and presenteeism, and (3) there is an association between patients' age and presenteeism.

\section{Materials and Methods}

The current study was based on data from medical records at the disposal of The Polish Social Insurance Institution stored in an electronic medical record of a network of 200 clinics functioning in large cities in Poland.

The indicator of presenteeism is the refusal to take sick leave in response to the doctor's advice. Such refusals are recorded and stored in a medical database. This indicator has the value of being more objective than self-reporting questionnaires, because patients refuse or accept to take a sick leave after being examined by a medical doctor and not just on the subjective sense of their own 
Table 1. Frequency distribution of the patients' age

\begin{tabular}{lc}
\hline Age, years & Patients, $n(\%)$ \\
\hline $19-24$ & $193(7.6)$ \\
$25-29$ & $541(21.4)$ \\
$30-34$ & $542(21.4)$ \\
$35-39$ & $467(18.5)$ \\
$40-44$ & $291(11.5)$ \\
$45-49$ & $160(6.3)$ \\
$50-54$ & $122(4.8)$ \\
$55-59$ & $127(5.0)$ \\
$60-64$ & $86(3.4)$ \\
\hline Total & $2,529(100)$ \\
\hline
\end{tabular}

health status which can lead to the decision of staying at work despite a worse health condition.

The current study is based on data of 2,529 patients aged 19-64 years $($ mean $=36.40, \mathrm{SD}=10.18)$ who were professionally active, either employed or running their own businesses in 31 different cities around the country, and were diagnosed with communicable diseases after a medical examination.

The database included data on 1,439 women ( $56.9 \%$ of the total sample) and 1,090 men (43.1\% of the total sample). They visited 1 of 150 outpatient clinics in Poland operated by Lux Med Ltd. in the period between October 20, 2014, and December 31, 2015. The clinics in this study all used the same unified electronic medical record system with the same method of data input on the sanction of sick leave. Patients were reimbursed via various methods of payment (national or private health insurance and out of pocket), but individual information was not provided. Each record included information about the age, gender, and diagnosis in terms of ICD-10 chapter, diagnosis in terms of ICD-10 code, and the patient's response to the doctor's recommendation on sick leave (coded dichotomically as either complying or refusing).

The statistical analysis consisted of appropriate statistical tests and effect size measures. Statistical tests were used to verify statistical significance, i.e., to verify whether associations exist and effect size measures were used to measure their strength. Statistically significant results are those which are unlikely to be results produced by chance.

Associations between refusals to take sick leave and the patients' age, gender, diagnosis in terms of ICD-10 main categories and detailed codes were tested with the use of Pearson's $\chi^{2}$ test of independence. The analysis was supplemented with Cramer's $V$ effect size measure. Cramer's $V$ effect size measure is a measure of the association between 2 categorical variables and it yields values between 0 and 1 (inclusive). These boundaries create an advantage over OR because the values of Cramer's $V$ have direct guidelines which enable interpretation in terms of small, medium, and large effect size.

According to these guidelines, depending on the number of variable categories, a value of 0.10 indicates a small effect size, a value of 0.30 indicates a medium effect size, and a value of 0.50 indicates a large effect size. When each of the 2 analyzed variables has 2 categories, or 0.04 indicates a small effect size, 0.13 indicates a medium effect size and 0.22 indicates a large effect size if the mul-
Table 2. Frequency distribution of diagnoses in terms of ICD-10 chapters

\begin{tabular}{lr}
\hline ICD-10 diagnosis & Patients, $n(\%)$ \\
\hline Salmonellosis (A02) & $16(0.63)$ \\
Yersiniosis (A04.6) & $1(0.04)$ \\
Giardiasis (A07.1) & $3(0.12)$ \\
Tuberculosis (A15-A19) & $14(0.55)$ \\
Brucellosis (A23) & $1(0.04)$ \\
Pertussis (A37) & $56(2.21)$ \\
Syphilis (A50-A53) & $9(0.36)$ \\
Lyme neuroborreliosis (A69.2) & $58(2.29)$ \\
Chlamydia infections (A74) & $22(0.87)$ \\
Dengue (A90) & $1(0.04)$ \\
Chikungunya (A92) & $1(0.04)$ \\
\hline Certain infectious and parasitic diseases & \\
$\quad$ A00-A99) in total & $182(7.20)$ \\
Measles (B05) & $2(0.08)$ \\
Rubella (B06) & $9(0.36)$ \\
Acute hepatitis C (B17.1) & $3(0.12)$ \\
Chronic viral hepatitis C (B18.2) & $37(1.46)$ \\
HIV infection (B20-B24) & $3(0.12)$ \\
Mumps (B26) & $14(0.55)$ \\
Malaria (B52) & $1(0.04)$ \\
Toxoplasmosis (B58) & $5(0.20)$ \\
Certain infectious and parasitic diseases & \\
$\quad$ B00-B99) in total & $74(2.93)$ \\
Influenza (J10-J11) & $2,273(89.88)$ \\
\hline
\end{tabular}

tiplication of the numbers of categories minus 1 in each of the 2 variables is equal to or greater than 5 .

The results of the analysis of relationships between the length of sick leave (expressed as the number of days) performed with the use of linear regression analysis on data acquired from patients who decided to accept sick leave were further used to estimate the possible length of sick leave in the patients who refused to take it. This allowed us to consider the scope of absenteeism that would have to be taken into account if the patients made a different decision and accepted sick leave.

The methodology used in this paper duplicates the calculations presented by Juszczyk et al. [9], which allowed us to compare the results of the 2 studies.

\section{Results}

\section{Sample Characteristics}

In most cases, the patients' age was between 25 and 34 years. Table 1 presents the frequency distribution of the patients' age. Table 2 provides the frequency distribution for diagnoses in terms of ICD-10 diagnosis. The majority of cases were diagnosed with influenza (J10-J11), which is an acute medical condition. The results in Table 2 support hypothesis $\mathrm{H} 1$. 
Fig. 1. Association between refusal to take sick leave and the patient's age.

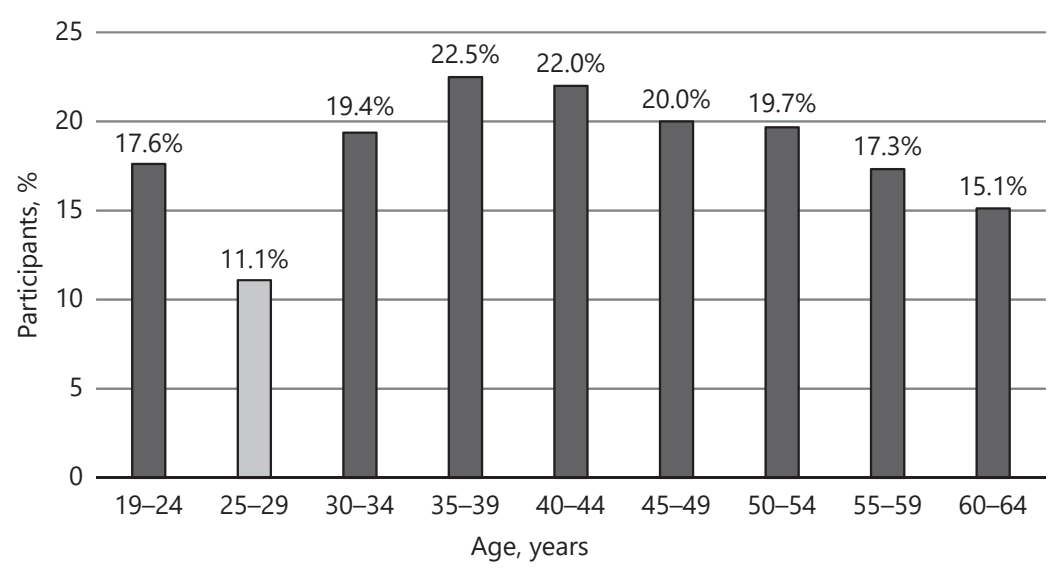

Table 3. Frequency distribution of sick leave refusals in the group of women and in the group of men

\begin{tabular}{lrrr}
\hline Sick leave & \multicolumn{1}{l}{ Women } & Men & \multicolumn{1}{l}{ Total } \\
\hline Compliance & $1,223(85.0)$ & $847(77.7)$ & $2,070(81.9)$ \\
Refusal & $216(15.0)$ & $243(22.3)$ & $459(18.1)$ \\
Total & $1,439(100)$ & $1,090(100)$ & $2,529(100)$
\end{tabular}

Values are presented as numbers (\%).

Refusals in Response to Doctor's Recommendations on Sick Leave

In the group of 2,529 patients, there were 459 patients (18.1\%) who refused to take sick leave after being diagnosed with a communicable disease. The remaining 2,070 patients $(81.9 \%)$ were on sick leave for a period lasting from 1 to 34 days $($ mean $=5.12, \mathrm{SD}=3.32$ ). Table 3 shows the frequency distribution of the sick leave refusals in the group of women and in the group of men.

On the basis of the $\chi^{2}$ independent test, the relationship between gender and sick leave refusal was found to be statistically significant $\left(\chi^{2}[1]=22.15, p<0.001\right)$. This result supports a hypothesis on gender differences in presenteeism. The number of patients who decided to refuse to take sick leave was slightly higher in the group of men than in the group of women. However, the effect size in terms of Cramer's $V$ coefficient was weak $(V=0.09, p<$ $0.001)$.

Also, on the basis of the $\chi^{2}$ independent test, the relationship between the participants' age and sick leave refusal was found to be statistically significant $\left(\chi^{2}[8]=\right.$

Refusal to Take a Sick Leave and

Presenteeism
$28.68, p<0.001)$. This result supports our hypothesis on the association between age and presenteesim. The number of patients who decided to refuse to take a sick leave was lower in the group of patients aged 25-29 years (Fig. 1). The effect size in terms of Cramer's $V$ coefficient was moderate $(V=0.11, p<0.001)$.

We also found a statistically significant relationship between diagnoses in terms of ICD-10 chapters and the refusal to take a sick leave $\left(\chi^{2}[2]=6.420, p<0.05\right)$. The effect size in terms of Cramer's $V$ measure was weak $(V=0.05, p<0.001)$. Figure 2 presents the frequency distribution for refusals in the groups of diseases. The categories were sorted from the one associated with the greatest number of refusals to the one associated with the least number of refusals. The refusals were most prevalent in cases with diagnoses from group $\mathrm{A}$ and least prevalent in group $\mathrm{B}$. The association between diagnosis in terms of ICD-10 codes and sick leave refusal was also statistically significant $\left(\chi^{2}[24]=54.596, p<\right.$ $0.001)$. The effect was strong $(\mathrm{V}=0.15, p<0.001)$. Half of the sample decided to refuse after being diagnosed with chlamydia or HIV infection, $30.4 \%$ of patients with pertussis, $26.9 \%$ with influenza, and $6.3 \%$ of those with salmonellosis.

\section{Estimation of the Length of Sick Leave}

The analysis of the relationship between the length of sick leave and the patients' age and gender was performed in a sample of 2,070 patients who accepted medical exemption. Linear regression analysis was used. The patients' age and gender were analyzed as predictors. The acquired regression coefficients are presented in Table 4. 
Fig. 2. Association between refusal to take sick leave and diagnosis in terms of ICD-10 diagnostic group.

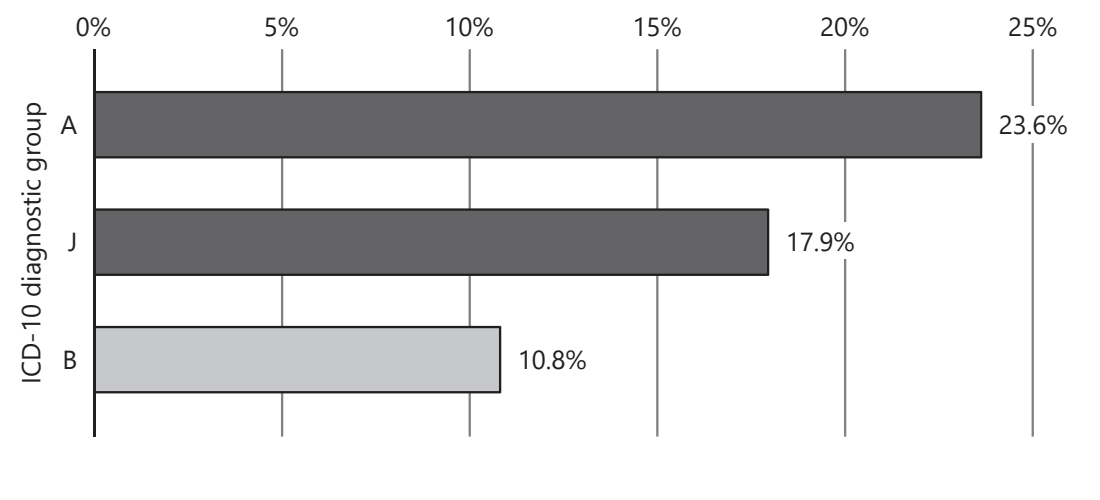

Table 4. Results of the linear regression analysis

\begin{tabular}{lrrrl}
\hline Predictor & \multicolumn{1}{l}{$\mathrm{B}$} & $\beta$ & \multicolumn{1}{l}{$t$} & \multicolumn{1}{l}{$p$} \\
\hline Constant & 3.65 & & 13.63 & 0.001 \\
Age & 0.04 & 0.13 & 6.16 & 0.001 \\
Gender & -0.26 & -0.04 & -1.78 & 0.076 \\
\hline
\end{tabular}

$B$, unstandardized regression coefficient; $\beta$, standardized regression coefficient; $t$, statistical test for predictor significance; $p$, statistical significance.

Table 5. Results of the linear regression analysis in the model in which age was analyzed

\begin{tabular}{lllrl}
\hline Predictor & B & $\beta$ & \multicolumn{1}{c}{$t$} & \multicolumn{1}{l}{$p$} \\
\hline Constant & 3.58 & & 13.51 & 0.001 \\
Age & 0.04 & 0.13 & 6.05 & 0.001 \\
\hline
\end{tabular}

$\mathrm{B}$, unstandardized regression coefficient; $\beta$, standardized regression coefficient; $t$, statistical test for predictor significance; $p$, statistical significance.

The results of the linear regression analysis performed in the model in which gender and age were analyzed as predictors of the length of sick leave (expressed in working days) in the group of patients who decided to comply with the doctor's recommendation and take sick leave. The analyzed model was statistically significant $(\mathrm{F}[2,2,067]=$ 19.90, $p<0.001$ ).

The relationships between the patients' age and the length of sick leave were statistically significant. The length of sick leave correlated positively with the patients' age. The older the patients were, the longer the periods of sick leave were. However, the relationship between the gender of the patients diagnosed with communicable diseases and the length of sick leave was statistically insignificant. In the final model, only the patients' age was used as a predictor. Table 5 presents the regression coefficients. The regression coefficients acquired in the final model were used to estimate the possible length of sick leave in the group of patients who refused to take it. The equation based on the unstandardized regression coefficients was:

Length of sick leave $($ in days $)=0.04 \times$ patient's age + 3.58 .

In the equation, gender is to be coded as 0 in the case of women and 1 in the case of men.

The gender and age range for patients who refused to take sick leave were substituted for the equation acquired in patients who decided to agree to take the leave. The estimated number of days spent on sick leave in the group of patients diagnosed with communicable diseases and refused to take it, assuming they made a different decision and complied to it, was in the range of 4-6 days (mean = $5.07, \mathrm{SD}=0.39$ ).

\section{Discussion}

In the current study, the percentage of professionally active people who decided not to take a break when they are not healthy and refuse to take sick leave upon being examined by a doctor and receiving a diagnosis of a communicable disease is $18.1 \%$. The estimation was based on the medical records of 2,529 patients. Assuming that the size of the adult population is $31,500,000$ the statistical error of the estimation is $2 \%$. The presenteeism rate in 
the current study is lower than the estimation based on the general sample, which was $27.4 \%$ [9]. When diagnosed with an infectious disease, fewer workers decided to be professionally active despite being ill, compared to the number of workers who refused to take sick leave in general, regardless of the diagnosis. It is possible that patients take the possibility of infecting others into consideration; however, this issue needs further research. As proposed in our hypotheses, we did observe a relationship between the type of diagnosis and presenteeism. The majority of cases in the current study were employees diagnosed with influenza. Its infectiousness was probably obvious to employees who decided to continue working despite their current medical condition. It is also an acute disease; however, many patients may think about it as being temporary and therefore decide to continue working [19].

In other countries, the prevalence of presenteeism seems to be even higher. In the UK, $90 \%$ of British social workers stated that they go to work when feeling ill at least once a year [20]. The prevalence of presenteeism in Montenegro, Slovenia, Malta, Denmark, and Sweden is above $50 \%$. In Italy, Portugal, and Bulgaria, it is estimated to be between 23 and $25 \%$ [21].

The results from the general sample [9] revealed that the number of patients who decided to refuse to take sick leave was also slightly higher in men than in women. In the current study, as hypothesized, there was a statistically significant association between presenteeism and gender, but it was weak. Research projects that investigated the relationship between presenteeism and gender also yielded contradictory results. Some showed that more women are involved in presenteeism than men [10], while others indicated that presenteeism is more frequent in men than in women [22]. The samples from the general population included patients diagnosed with diseases which occur with the same prevalence in women and men, patients diagnosed with diseases which occur more frequently in women or men, and patients diagnosed with diseases which are gender specific. The sample composition of each sample in terms of the occurrence of a gender-specific diagnosis may bias the results and it seems that there is a need to control it in future research.

Presenteeism was least prevalent in the patients aged 25-29 years, which supported our hypothesis. In this study and the earlier one [9], periods of sick leave were longer in older patients. The age of patients seems to be a more common factor affecting presenteeism than the gender of the patients.

Refusal to Take a Sick Leave and Presenteeism
The level of generality of diagnostic codes is also a very important issue which can lead to different conclusions. In the current study, when looking at specific codes and comparing them to broader diagnostic categories in the chapters of the ICD-10, one can see that the estimated presenteeism in the case of acute upper respiratory infections (chapter J) can be interpreted as more or less prevalent depending on the reference background. It seems to be more prevalent compared to other diseases when using more general chapter codes than when using more specific ICD-10 diagnostic categories. Using more general diagnostic categories means that more detailed categories are combined, and one looks at the same phenomenon from a different perspective. In the results acquired from the general sample, specific ICD-10 codes revealed that acute sinusitis, acute pharyngitis, acute tonsillitis, and acute laryngitis and tracheitis, consequences of bacterial or virus infections and possibly contagious, were found to be the most frequent categories in which presenteeism occurred.

Possible predictors of being professionally active in spite of being ill can be divided into the following 5 categories: personality traits, attitudes, organizational policies, job design features, and culture. Conscientiousness was found to be positively related to presenteeism [23]. Higher self-efficacy translates to a higher level of presenteeism [24]. Identification with professional activity strengthens presenteeism [25]. The policy of taking disciplinary action after repeated absences can induce presenteeism [12]. Downsizing is another possible reason in the group of temporary employees, who are most likely to lose their jobs [26]. Having no permanent employment itself is also a predictor of presenteeism [27]. It was also tested as a predictor of presenteeism in longitudinal research [28]. An attempt to explain which of these phenomena may have a dominant impact on presenteeism in Poland still needs further research. Another important factor that can have an impact on presenteeism is workers' wages. Aronsson et al. [11] found that higher wages are negatively correlated with absenteeism. Hansen and Andersen [29] did not confirm this conclusion. Since the Polish accession to the European Union, the average wage in the national economy has almost doubled (in 2004 it was PLN 2,290, and in the third quarter of 2017 it was PLN 4,256), and the minimum wage nearly tripled (in 2004 it was PLN 824, and in 2017 it was PLN 2,100). It is possible that this dynamic of growth had a considerably low effect on the presenteeism rate when compared to the results acquired in other countries with higher wages but a lower growth.

Med Princ Pract 2020;29:134-141 139 
The calculations made in the present paper allowed for a conclusion that if the patients decided to take sick leave then they would be absent from work for a period of 4-6 days (mean $=5$ ). Taking the type of health problem involved into account and the possibility of infecting others, it is difficult to see how presenteeism may be beneficial from the productivity point of view. It is the precise estimation of costs that could be reduced by cutting down on presenteeism in the case of communicable diseases that is difficult, although there is no doubt that it would be profitable in the long run. The difficulty, in this case, is the fact that infection precedes the onset of symptoms. The best possible solution would be to conduct a study in which effective psychosocial intervention or organizational policy leading to lowering of the presenteeism rates would be assessed in terms of its effectiveness and in terms of a cost reduction attributable to absences at work, taking into account that there is a possibility of replacement of one professional by another, which also affects costs.

An estimation of the precise costs of presenteeism would involve using a form of self-estimation of productivity loss. There are at least 3 approaches: estimation of productivity loss in hours (alternatively, respondents may be asked to estimate the extra hours that would be needed to compensate for inefficient hours), estimation of the perceived percentage loss (the most widely used), and a comparison between the productivity loss obtained from an individual and that obtained from a healthy colleague in a similar role [30]. Having obtained a measure of productivity loss and taking into account the costs and benefits of working in full health, one can convert the measure into a monetary estimate. Combining self-measure techniques and medical records would make this estimation more accurate.

\section{Conclusion}

The prevalence of presenteeism in the case of communicable diseases acquired in the current study in Poland is lower than in the general population. However, the most frequent diagnoses in the analyzed group of patients who decided to work despite their medical condition was influenza, which is highly infectiousness. Its negative impact on productivity may be significant. The patients who start their professional career being 25-29 year olds were the least willing to work being ill.

\section{References}

1 Darr W, Johns G. Work strain, health, and absenteeism: A meta-analysis. J Occup Health Psychol. 2008;13(4):293-318.

2 Johns G, Miraglia M. The reliability, validity, and accuracy of self-reported absenteeism from work: A meta-analysis. J Occup Health Psychol. 2015;20(1):1-14.

3 Vargas-Prada S, Demou E, Lalloo D, AvilaPalencia I, Sanati KA, Sampere M, et al. Effectiveness of very early workplace interventions to reduce sickness absence: a systematic review of the literature and meta-analysis. Scand J Work Environ Health. 2016;42(4): 261-72.

4 Skåtun JD. Take some days off, why don't you? Endogenous sick leave and pay. J Health Econ. 2003;22:379-402.

5 Burton WN, Pransky G, Conti DJ, Chen CY, Edington DW. The association of medical conditions and presenteeism. J Occup Environ Med. 2004;46:S38-45.

6 Collins JJ, Baase CM, Sharda CE, Ozminkowski RJ, Nicholson S, Billotti GM, et al. The assessment of chronic health conditions on work performance, absence, and total economic impact for employers. J Occup Environ Med. 2005;47(6):547-57.
7 Goetzel RZ, Long SR, Ozminkowski RJ, Hawkins K, Wang S, Lynch W. Health, absence, disability, and presenteeism cost estimates of certain physical and mental health conditions affecting U.S. employees. J Occup Environ Med. 2004;46(4):398-412.

8 Hemp P. Presenteeism: at work - but out of it. Harv Bus Rev. 2004;82:49-58.

9 Juszczyk G, Czerw A, Augustynowicz A, Banaś T, Mikos M, Religioni U, et al. Refusal to take a sick leave as an estimate of the phenomenon of presenteeism in Poland. Oncotarget. 2018 Jun;9(46):28176-84.

10 Aronsson G, Gustafsson K. Sickness presenteeism: prevalence, attendance-pressure factors, and an outline of a model for research. J Occup Environ Med. 2005;47(9):958-66.

11 Aronsson G, Gustafsson K, Dallner M. Sick but yet at work. An empirical study of sickness presenteeism. J Epidemiol Community Health. 2000;54(7):502-9.

12 Grinyer A, Singleton V. Sickness absence as risk-taking behaviour: A study of organizational and cultural factors in the public sector. Health Risk Soc. 2000;2(1):7-21.

13 Caverley N, Cunningham JB, MacGregor JN. Sickness presenteeism, sickness absenteeism, and health following restructuring in a public service organization. J Manage Stud. 2007; 44(2):304-19.
14 Demerouti E, Le Blanc PM, Bakker AB, Schaufeli WB, Hox J. Present but sick: A three-wave study on job demands, presenteeism and burnout. Career Dev Int. 2009;14(1): 50-68.

15 Hackett RD, Bycio P, Guion RM. Absenteeism among nurses: an idiographic-longitudinal analysis. Acad Manage J. 1989;32:424-53.

16 Adelman JU, Sharfman M, Johnson R, Miler D, Clements B, Gayla Pait D, et al. Impact of oral sumatriptan on workplace productivity, health-related quality of life, health care use, and patient satisfaction with medication in nurses with migraine. Am J Manag Care. 1996; 10:1407-16.

17 Johns G. Presenteeism in the workplace: A review and research agenda. J Organ Behav. 2010;31(4):519-42.

18 European Commision. Commission Implementing Decision (EU) 2018/945 on the communicable diseases and related special health issues to be covered by epidemiological surveillance as well as relevant case definitions. Official J Eur Union. 2018; 61:170.

19 Cohen J. Statistical power analysis for the behavioral sciences. New York: Lawrence Earlbaum Associates; 1988. 
20 Ravalier JM. The influence of work engagement in social workers in England. Occup Med (Lond). 2018;68(4):399-404.

21 Garrow V. Presenteeism. A review of current thinking. Brighton: Institute for Employment Studies; 2016.

22 Voss M, Floderus B, Diderichsen F. How do job characteristics, family situation, domestic work, and lifestyle factors relate to sickness absence? A study based on Sweden Post. J Occup Environ Med. 2004;46(11):1134-43.

23 Yildiz H, Yildiz B, Zehir C, Altindağ E, Moloğlu V, Kitapçi H. Impact on presenteeism of the conscientiousness trait: a health sector case study. Soc Behav Pers. 2017;45(3): 399-411.
24 Palo S, Pati S. The determinants of sickness presenteeism. Indian J Ind Relat. 2013;49: 256-69.

25 Van Maanen J, Barley SR. Occupational communities: culture and control in organizations. Res Organ Behav. 1984;6:287-365.

26 Vahtera J, Kivimaki M, Pentti J, Linna A, Virtanen M, Virtanen P, et al. Organisational downsizing, sickness absence, and mortality: 10-town prospective cohort study. BMJ. 2004; 328(7439):555.

27 Gimeno D, Benavides FG, Amick BC, Benach J, Martínez JM. Psychosocial factors and work related sickness absence among permanent and non-permanent employees. J Epidemiol Community Health. 2004;58(10):870-6.
28 Virtanen M, Kivimaki M, Elovainio J, Vahtera J, Ferrie JE. From insecure to secure employment: changes in work, health, health related behaviours, and sickness absence. Occup Environ Med. 2003;60(12):948-53.

29 Hansen CD, Andersen JH. Going ill to workwhat personal circumstances, attitudes and workrelated factors are associated with sickness presenteeism? Soc Sci Med. 2008;67(6): 956-64.

30 Mattke S, Balakrishnan A, Bergamo G, Newberry SJ. A review of methods to measure health-related productivity loss. Am J Manag Care. 2007;13:211-7. 